\title{
Design and Development of a Drone for Spraying Pesticides, Fertilizers and Disinfectants
}

\author{
Karan Kumar Shaw ${ }^{1}$, Vimalkumar R. ${ }^{2}$ \\ ${ }^{1,2}$ UG Students, Batch-2021, Department of Aerospace Engineering, SRM Institute of Science and Technology, \\ Kattankulathur, Chengalpattu District, Tamil Nadu-603203, India.
}

\begin{abstract}
There are too many technologies involved in today's Agriculture, out of which spraying pesticides using drones is one of the emerging technologies. Manual pesticide spraying causes many harmful side effects to the personnel involved in the spraying process. The Exposure effects can range from mild skin irritation to birth defects, tumors, genetic changes, blood and nerve disorders, endocrine disruption, coma or death. The WHO (World Health Organization) estimated as one million cases of ill affected, when spraying the pesticides in the crop field manually. This paved the way to design a drone mounted with spraying mechanism having $12 \mathrm{~V}$ pump, 6 Litre storage capacity tank,4 nozzles to atomize in fine spray, an octocopter configuration frame, suitable landing frame, 8 Brushless Direct Current (BLDC) motors with suitable propellers to produce required thrust about 38.2 KG(at 100\% RPM) and suitable LithiumPolymer (LI-PO) battery of current capacity $22000 \mathrm{mAh}$ and 22.2 $\mathrm{V}$ to meet necessary current and voltage requirements. A First-Person View (FPV) camera and transmitter can also be fixed in the drone for monitoring the spraying process and also for checking pest attacks on plants. This pesticide spraying drone reduces the time, number of labor and cost of pesticide application. This type of drone can also be used to spray disinfectant liquids over buildings, water bodies and in highly populated areas by changing the flow discharge of the pump.
\end{abstract}

Key Words: DRONE, AGRICULTURE, PAYLOAD, SENSORS, PUMP, SPRAY

\section{INTRODUCTION}

The Indian Agricultural sector is the most important sector as it amounts to a staggering $18 \%$ of India's Gross Domestic Product (GDP) and also provides employment to $50 \%$ of the national human workforce. Our country is dependent on agriculture so much, has yet to tap into the real potential of agriculture, because of improper methods of monitoring crops and the irrigation patterns and the pesticides required to be applied. In India, there are over 35 drone start-ups that are working to raise the technological standards and reduce the prices of agricultural drones. This project aims to develop Unmanned Aerial Vehicle (UAV) for overcoming this problem and also spay large amounts of pesticides within smaller interval of time using Octocopter.

\subsection{Literature Survey}

Dongyan et al. (2015) [1] experimented on effective swath width and uniformity of droplet distribution over aerial spraying systems like M-18B and Thrush 510G.These agricultural planes flew at height of $5 \mathrm{~m}$ and $4 \mathrm{~m}$ respectively and with this experiment they reach to conclusion that flight height leads to the difference in swath width for M-18B \& Thrush 510G.
Huang et al. (2015) [2] made a low volume sprayer which is integrated into unmanned helicopters. The helicopter has a main rotor diameter of $3 \mathrm{~m}$ and a maximum payload of 22.7 $\mathrm{kg}$. It used to require at least one gallon of gas for every 45 minutes. This study paved the way in developing UAV aerial application systems for crop production with higher target rate and larger VMD droplet size.

Yallappa et al. (2017) [3] developed an hexacopter with 6 BLDC motors and two LiPo batteries of 6 cells- $8000 \mathrm{mAh}$. Their study also involves performance evaluation on discharge and pressure of spray liquid, spray liquid loss and determination of droplet size and density. Through their project, they finally made a drone capable of carrying $5.5 \mathrm{~L}$ of liquid with an endurance time of 16 min.

Kurkute et al. (2018) [4] worked on quadcopter UAV and its spraying mechanism using simple cost-effective equipment. The universal sprayer system is used to spray for both liquid and solid content. In their research, they have also compared different controllers needed for agricultural purposes and concluded that quadcopter system with Atmega644PA is the most suitable due to its efficient implementation.

Rahul Desale et al. (2019) [5] described an architecture based on UAV that could be employed for agricultural applications. Their UAV was designed not only for spraying but also for monitoring agricultural fields with the use of cameras and GPS. Their design was optimized for cost and weight. They used a microcontroller kk 2.1.5 which has inbuilt firmware.

Prof. B. Balaji et al. (2018) [6] developed an hexacopter UAV with the purpose of spraying pesticides as well as crop and environment monitoring using Raspberry $\mathrm{Pi}$ that run on python language. Their UAV also contains multiple sensors like DH11, LDR, Water Level Monitoring sensors. From this experiment, they finally concluded that with proper implementation of UAVs in the agricultural field almost $20 \%$ $90 \%$ savings in terms of water, chemical maltreatments and labor can be expected.

\section{DESIGN AND WORKING OF OCTOCOPTER}

To Design an octocopter first we have to Estimate our payload, then with respect the weight of the payload motor, Propeller, Electronic Speed Controller, Pump, First Person View camera and video transmitter has to be selected. Battery has to be selected by knowing the current and voltage requirements of the components. Then the thrust requirement has to be calculated and finally the frame of the copter has to be designed by determining required arm number, arm length and application of payload. 


\subsection{Payload Estimation}

The weight of the payload is calculated by taking the weight of the liquid (pesticide or disinfectant), storage tank of 6 Litre capacity, pump and nozzles.

Table -1: Payload Data

\begin{tabular}{|c|c|}
\hline PARTS & WEIGHT (grams) \\
\hline 6 Litre liquid & 6000 \\
\hline 6 Litre liquid tank & 250 \\
\hline pump & 200 \\
\hline Nozzle & 300 \\
\hline Total & 6750 \\
\hline
\end{tabular}

\subsection{Construction}

The prefix octo-copter implies ("octo" =eight), is a drone configuration where there are eight arms. The main frame is made of carbon fiber composite material with each arm length of $492 \mathrm{~mm}$. At each free end of the arm, a motor will be fixed and propeller will be mechanically coupled to the motor. For all eight motors the output side of an ESC will be connected and the input side of the Electronic Speed Controller (ESC) will be connected to the flight controller. The other input of the ESC will be connected to the power distribution board where the power supply is provided by the Li-Po battery. In a similar fashion all the other ESC's, motors and propellers are connected. A receiver will be connected to the Flight controller to receive signals from the transmitter. An FPV camera and a suitable transmitter connected each other is connected to the flight controller. The storage tank of dimensions $200 \times 300 \times 110 \mathrm{~mm}$ is mechanically coupled to the frame, the bottom of the tank will have a slope so that the entire tank gets drained completely. A plastic tube of 1.3 meters length and four nozzles are fixed at $45 \mathrm{~cm}$ between each other. A pump is powered from a power distribution board, the inlet of the pump is connected to the storage tank and the outlet is connected to the plastic tube where nozzles are fixed. The landing frame of height $300 \mathrm{~mm}$ is connected to the main frame so that the landing of the drone will be safe and the storage tank will not touch the ground.

\subsection{Working}

The signals will be transmitted from Transmitter and it will be received by the Receiver in the drone. From the receiver the signal goes to the Flight controller where the signal will be processed with accelerometer and gyroscope sensors. The processed signal will be sent to the ESC, which allows the specific amount of current to the motor based on the signal it receives. The propellers are mechanically coupled to the motors so that they rotate and produce thrust. The FPV camera takes current supply from the flight controller and it records the video, the video signals will be processed by the transmitter and it will be received by the receiver in ground. The pump takes current supply from the Li-Po battery and pressurizes the liquid from the storage tank then the pressurized liquid flows through the pipeline and enters the nozzle then gets sprayed. The flow rate of the pump can be controlled by varying the input current which can be controlled from the transmitter.

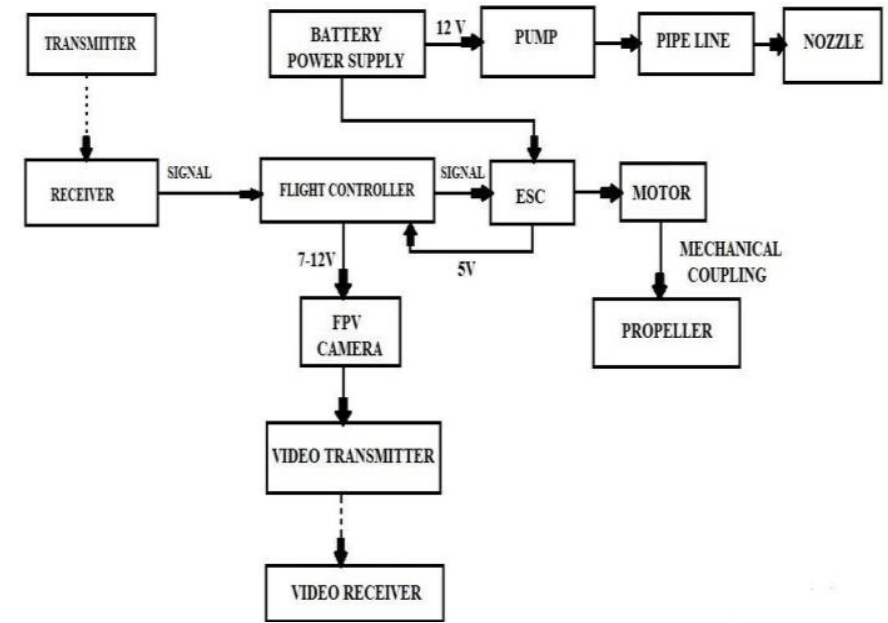

Fig -1: Block diagram of working process

\subsection{Components Used}

\subsubsection{Motor}

Outer runner BLDC motors in which there are no brushes, they have a permanent magnet. The RPM of the motor can be controlled by varying the input current. This motor TMOTOR MN 7005 KV115 and P24x7.2F propeller produces a maximum thrust of 4783 grams.

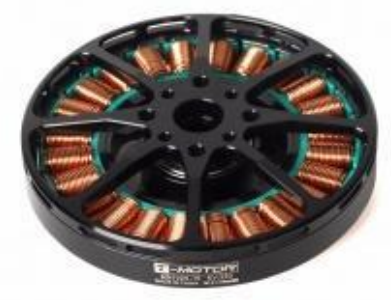

Fig -2: T-MOTOR MN 7005 KV115

\subsubsection{Propeller}

The propeller is of 24 inches length and has 7.2 inches pitch. It is made up of carbon fiber which possesses high strength to weight ratio when compared to the propellers made up of plastics.

Fig -3: T-MOTOR Propeller P24X7.2CF

\subsubsection{ESC}

It stands for Electronic Speed Controller and it is used to vary the Revolution Per Minute (RPM) of the motor. 60A rated ESC is used as per the motor and battery specifications. 


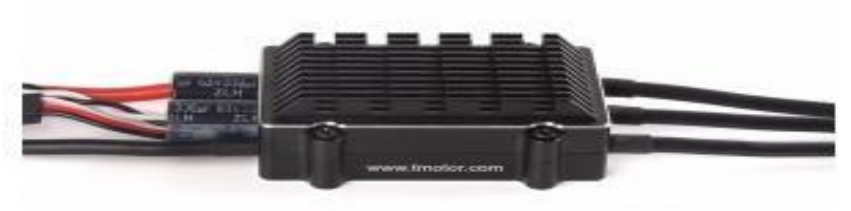

Fig -4: Flame 60A HV

\subsubsection{Battery}

The battery that can be used is a Li-Po battery of 22000mAh capacity and $22.2 \mathrm{~V}$. In this battery six Li-Po cells are connected in series $(6 \times 3.7=22.2 \mathrm{~V})$.

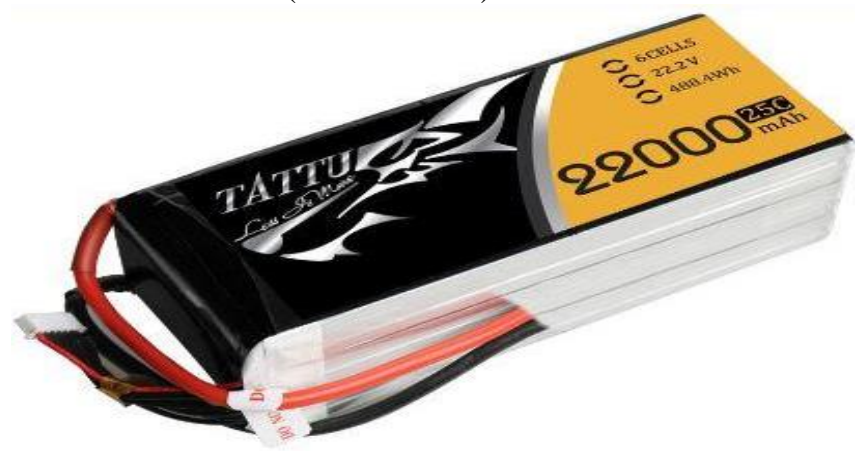

Fig -5: Li-Po battery

\subsubsection{Flight Controller}

The flight controller helps in the maneuvering operations and also it provides Auto level function. The accelerometer and gyroscope sensors in the Flight controller processes the signals from the receiver and gives the output to the ESC. The KK 2.1.5 Flight controller board can be used in the drone as it has inbuilt firmware. The features of this Flight controller board are much easier for calibration. It uses ATMEL Mega 644PA 8-bit AVR RISC-based microcontroller with 64K of memory.

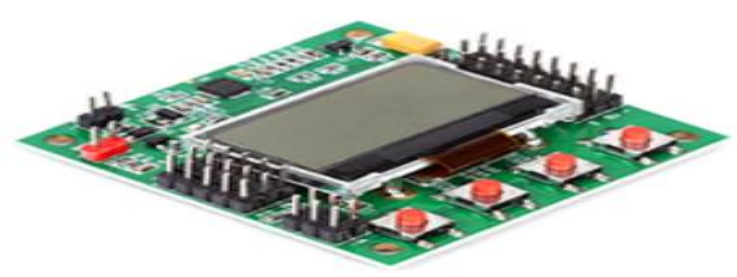

Fig -6: KK 2.1.5 Multi-Rotor LCD Flight controller

\subsubsection{Radio Transmitter and Receiver}

The Transmitter and receiver used are FlySky CT6B 2.4Ghz $6 \mathrm{CH}$ and FS-R6B respectively. This combination provides a range of about 1000 meters. This Transmitter and receiver provide upto 6 channel options.

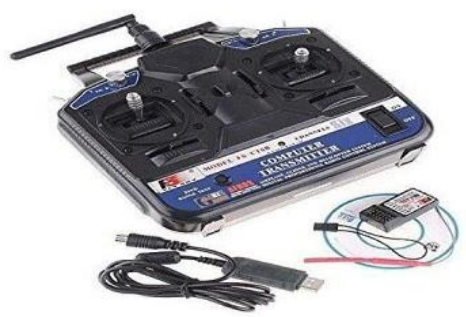

Fig -7: Fly sky CT6B 2.4 6CH Transmitter and FS-R6B Receiver

\subsubsection{FPV Camera and Transmitter}

The camera that can be used is HD FPV camera 1200 TVL, it has $2.8 \mathrm{~mm}$ Lens, auto/color/ black \& white Day and night format.TS5828 32CH mini transmitter can be connected to the camera for transmission of video signals to receiver at ground.
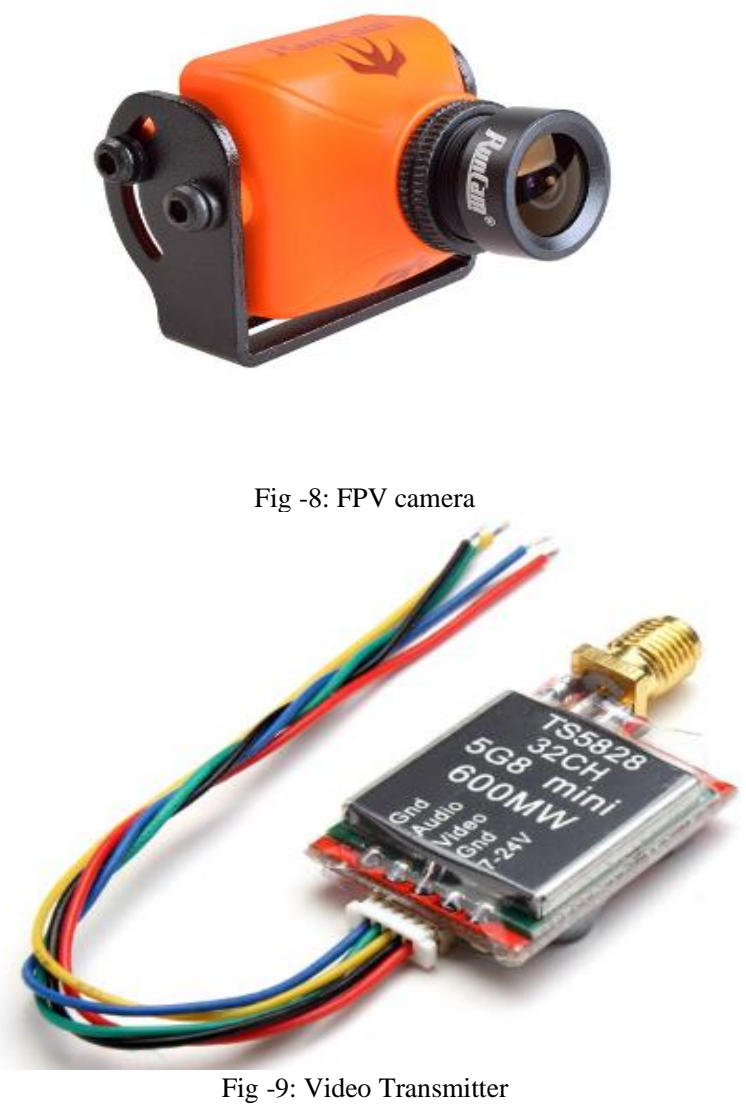

5.8G UVC receiver is used to receive the video signals. It can be connected to the android mobile which has installed the GO FPV application in it. 


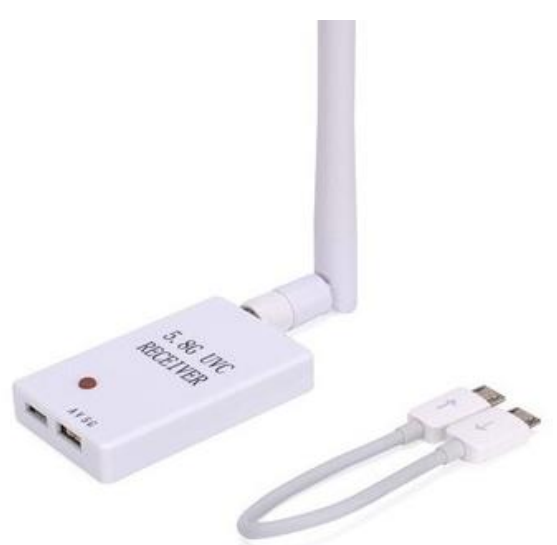

Fig -10: 5.8 UVC OTG Receiver

\subsubsection{Pump and Nozzle}

To pressurize the liquid a $12 \mathrm{~V}$ DC water pump can be used which has $2.5 \mathrm{~L} / \mathrm{min}$ capacity can be used. Then the pressurized liquid enters the nozzle and gets sprayed. The nozzle that can be used is a flat fan type for spraying the liquid. Four nozzles are connected with ducts and they are palace at $45 \mathrm{~cm}$ distance between each other.
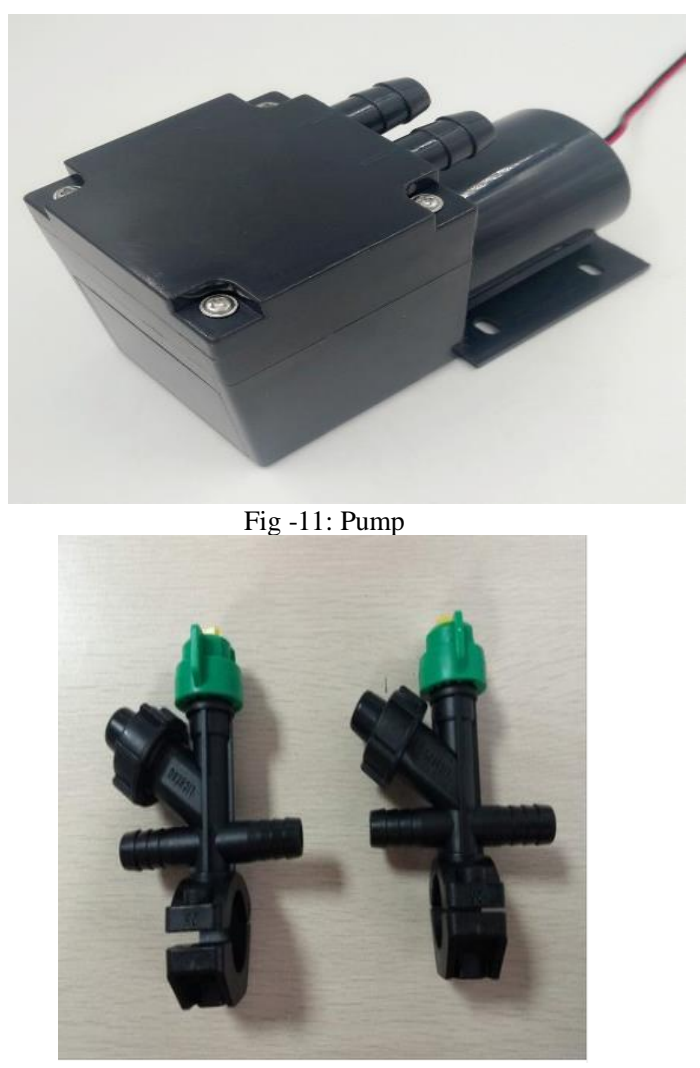

Fig -12: Flat fan Nozzle

\subsection{Weight Build-up}

Table -2: Components Weight Datasheet

\begin{tabular}{|c|c|}
\hline PARTS & WEIGHT (grams) \\
\hline Frame & 2000 (Approx.) \\
\hline Battery & 1386 \\
\hline Motor $(8)$ & 1504 \\
\hline ESC (8) + power distributor & 588 \\
\hline Propeller (8) & 448 \\
\hline Flight controller & 20 \\
\hline TOTAL & 5946 \\
\hline
\end{tabular}

The overall weight of the drone is calculated by adding the total weight of components and the weight of payload.

Overall weight $=$ Payload + Weight of components

$$
\begin{aligned}
& =6750+5946 \\
& =12,696 \text { grams(approx.) }
\end{aligned}
$$

\subsection{Thrust Calculation}

Thrust developed at 100\% RPM can be three times larger than the total weight of the drone so that the drone has better maneuverability and the drone can climb higher altitudes with higher rate of climb.

Thrust produced by one propeller with one motor $=4783$ grams

Total thrust produced $=8 \times 4783=38264$ grams

Thrust to weight Ratio $=$ Thrust produced $/$ total weight of drone

$$
\begin{aligned}
& =38264 / 12696 \\
& =3.01: 1
\end{aligned}
$$

\subsection{Battery Drain Time Calculation}

The Battery Drain time can be more than 10 minutes so that the drone drains the entire storage tank and it can be refilled for another serve. For a safer side, the battery drain time has to be calculated by considering the distance and time for the drone to return safely.

Table -3: Current Requirement Table

\begin{tabular}{|c|c|}
\hline COMPONENT & CURRENT REQUIRED (Amp) \\
\hline Motor & 120 \\
\hline Receiver & 0.1 \\
\hline Flight controller & 0.1 \\
\hline ESC $(8)$ & 0.8 \\
\hline Camera & 0.32 \\
\hline FPV transmitter & 0.31 \\
\hline pump & 5 \\
\hline TOTAL & 126.63 \\
\hline
\end{tabular}

Current output from battery $=22000 \mathrm{MAh}$

Total current consumption of all components $=126.63 \mathrm{~A}$

Battery endurance $=$ current output from battery/ Total current consumption of all components

$$
\begin{aligned}
& =22000 \mathrm{MAh} / 126.63 \mathrm{~A} \\
& =22 * 60 / 126.63 \mathrm{~A} \\
& =10.42 \quad \mathrm{MINS} .
\end{aligned}
$$

(AT $\quad 100 \%$

THROTTLE)

\subsection{Structural Description}

There is no such generic frame which is considered to be best among all the frames, however it depends on a number of factors like multi-rotor configuration, rotating moment and balancing, application, material, stiffness and components integration. Depending on size and dimensions of selected electronic components, we designed a base plate in the shape of irregular octagon such that all components placed on the Centre of Gravity. The dimensions in $\mathrm{mm}$ are briefly mention on figure 13. 


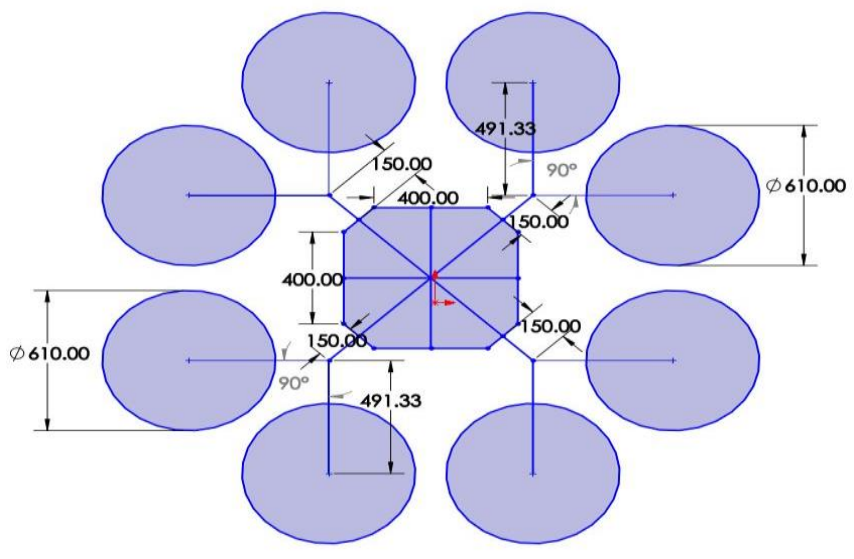

Fig -13: 2D Drawing

We decided to use shorter four "Y-shaped" arms rather than going for longer 8 straight arms as it could reduce weight. Moreover, the former configuration has more stable thrust balancing and smaller in size which make it easier to carry as compared to the latter. The dimensioning of arms was done maintaining the minimum gap of $5 \mathrm{~cm}$ between propellers and angles between two arms of $\mathrm{Y}$ is subjective to application of drone. The cross-section of the arms were hollow circular rods because it can withstand the same stress as that of solid rods. In torsion the stress profile is a linear relationship with diameter. Strength to weight ratio is better for a hollow pipe than a solid rod. This means a hollow cylinder is stronger than a rod of equal mass and the same material.

The selection of material is as crucial as selection of configuration of arms. The material plays a significant role in determining the drone's stability and efficient performance. The material for the arms was chosen as carbon fibre due to its properties like light weight and higher strength- to-weight ratio. However, the radio and antenna were connected properly to stop the radio signal interference by carbon fibers. The material for base plate was selected as Carbon/Kevlar composite due to its properties like high tensile strength, no shrinkage and embrittlement at extreme temperature, scratch and electrically resistant. The landing frame is made up of aluminium alloy owing to its light weight and yield strength. The cad model of our design as shown in figure 13 is made in SOLIDWORKS 20.

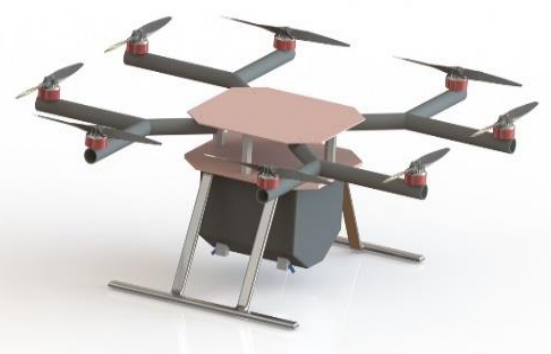

Fig -14: CAD Model of Drone
The tank being placed at the CG of the drone having capacity of 6 Litre having slanting bottom to prevent liquid stagnation during gravity feed. It is made up of Polyethylene which is the most common plastic tank material as it is relatively immune to damage to salt water. Any non-hazardous liquid can be used in the tanks for the purpose spraying.

\section{CONCLUSIONS}

In this paper we have described a design of a drone mounted spraying mechanism for Agricultural purpose and for spraying disinfectants. This method of spraying pesticides on Agricultural fields reduces the number of labours, time, cost and the risk involved to the personnel involved in spraying the liquids. This drone can also be used in spraying disinfectant liquids over buildings, water bodies and highly populated areas.

\section{FUTURE SCOPE}

$>$ Under the current COVID19 Pandemic situation, it can be used to sanitize large hotspots areas without actually going there in person.

$>$ Manual control can be changed into autonomous control with GPS technology and auto return home option.

$>$ With image processing techniques, the drone can be involved in surveillance to determine the pest attack on the plants, condition of ripening fruit.

\section{REFERENCES}

[1] Zhang Dongyan, Chen Liping, Zhang Ruirui, Xu gang, Lan Yubin, Wesley Clint Hoffmann, Wang Xiu, Xu Min, "Evaluating effective swath width and droplet distribution of aerial spraying systems on M18B and Thrush 510G airplanes", April 2015, Int J. Agric. \& Bio Eng, Vol 8 No.21.

[2] Huang, Y. Hoffmann, W.C. Lan, Y. Wu and Fritz, B.K, "Development of a spray system for an unmanned aerial vehicle platform”, Dec 2015, Applied Engineering in Agriculture, 25(6):803-809.

[3] Yallappa D., M. Veerangouda, Devanand Maski, Vijayakumar Palled and M. Bheemanna, "Development and Evaluation of Drone mounted sprayer for Pesticides Applications to crops.” Oct. 2017, Research Gate, Conference paper.

[4] S.R. Kurkute, B.D. Deore, Payal Kasar, Megha Bhamare, Mayuri Sahane, "Drones for Smart Agriculture: A Technical Report", April 2018, IJRET, ISSN: 2321-9653.

[5] Rahul Desale, Ashwin Chougule, Mahesh Choudhari, Vikrant Borhade, S.N. Teli, "Unmanned Aerial Vehicle for Pesticides Spraying" April 2019, IJSART, ISSN: 2395-1052.

[6] Prof. B.Balaji, Sai Kowshik Chennupati, Siva Radha Krishna Chilakalapudi, Rakesh Katuri, kowshik Mareedu, "Design of UAV (Drone) for Crops, Weather Monitoring and For Spraying Fertilizers and Pesticides.”, Dec 2018, IJRTI, ISSN: 2456-3315.

[7] Prof. P. Mone, Chavhan Priyanka Shivaji, Jagtap Komal Tanaji, Nimbalkar Aishwarya Satish, "Agriculture Drone for Spraying Fertilizers and Pesticides", Sept 2017, International Journal of Research Trends and Innovation, ISSN 2456-3315, Volume 2, Issue 6.

[8] F. G. Costa, J. Ueyama, T. Braun, G. Pessin, F. S. Osorio, P. A. Vargas, "The Use of Unmanned Aerial Vehicles and Wireless Sensor Network in Agriculture Applications", 2012, IEEE International Geoscience and Remote Sensing Symposium 2012.

[9] Spoorthi, S., Shadaksharappa, B., Suraj, S., Manasa, V.K.,"Freyr drone: Pesticide/fertilizers spraying drone-an agricultural approach.", 2017, IEEE 2nd International Conference on In Computing and Communications Technologies, pp. 252-255.

[10] G. Ristorto, F. Mazzetto, G. Guglieri, and F. Quagliotti, "Monitoring performances and cost estimation of multirotor unmanned aerial systems in precision farming." ,2015, International Conference on Unmanned Aircraft Systems (ICUAS), pp. 502-509. 